\title{
Searches for New Physics at Colliders
}

\author{
Giorgio Chiarelli
}

INFN Sezione di Pisa, Via F.Buonarroti 2, I-56127 Pisa

\begin{abstract}
In this paper I present the most recent results of the ongoing searches, mainly from Tevatron Collider experiments, for new physics beyond the Standard Model. While no signal has been seen so far, many analyses are reaching the point in which either a discovery will take place or strong limit on currently popular theories will be set.
\end{abstract}

\section{INTRODUCTION}

The standard model of elementary particles and fundamental interactions (from now on Standard Model or SM) has been very successfull to describe the subnuclear world. Given this succes one would wonder why should we look at any theory beyond the standard model (BSM) at all. There are several theory oriented answers to this question, from the hyerarchy problem to the stability of the Higgs sector, to the number of generations, just to name a few. In my view, however, most important are some hints of open questions. What is the nature of the Cold Dark Matter (now that we have an indirect cosmological proof of its existence). Why neutrinos have masses? Is there a unification of the coupling constants before the Planck scale? Finally, while the SM has been succesful we still do not have a way to marry quantum theory with gravity, let alone to unify gravity with the other interactions.

An optimal place to look for new phenomena is the energy frontier which, at the moment, is explored by the experiments H1 and ZEUS at the HERA collider at DESY near Hamburg [1], where proton and electron (or positron) collide at $\sqrt{s}=320 \mathrm{GeV} / \mathrm{c}^{2}$ and at the Tevatron (experiments CDF and D0) where proton and antiproton collide at the highest energy $(1.96 \mathrm{TeV})$ currently available [2]. In the near future that frontier will be throughly explored by the LHC, currently being built at CERN. Most of the results presented were obtained at the Tevatron where the goal of $1 \mathrm{fb}^{-1}$ delivered per experiment was exceeded in May 2005. Current scenarios predict between 4 and $8 \mathrm{fb}^{-1}$ delivered (per experiment) by 2009.

This exploration physics proceeds mainly through comparison of data with SM expectations. As New Physics (NP) is anything which is beyond the standard model, the best experimental way to look for it in a model independent way is to select observables that can be affected by NP and then compare measurements with SM expectations. It takes a lot of ingenuity but it also implies that in those searches we will be studying familiar physics objects. Hadronic jets, charged and neutral leptons are the basic tool. ${ }^{1}$

${ }^{1}$ In the following we will use MET for Missing Transverse Energy. 


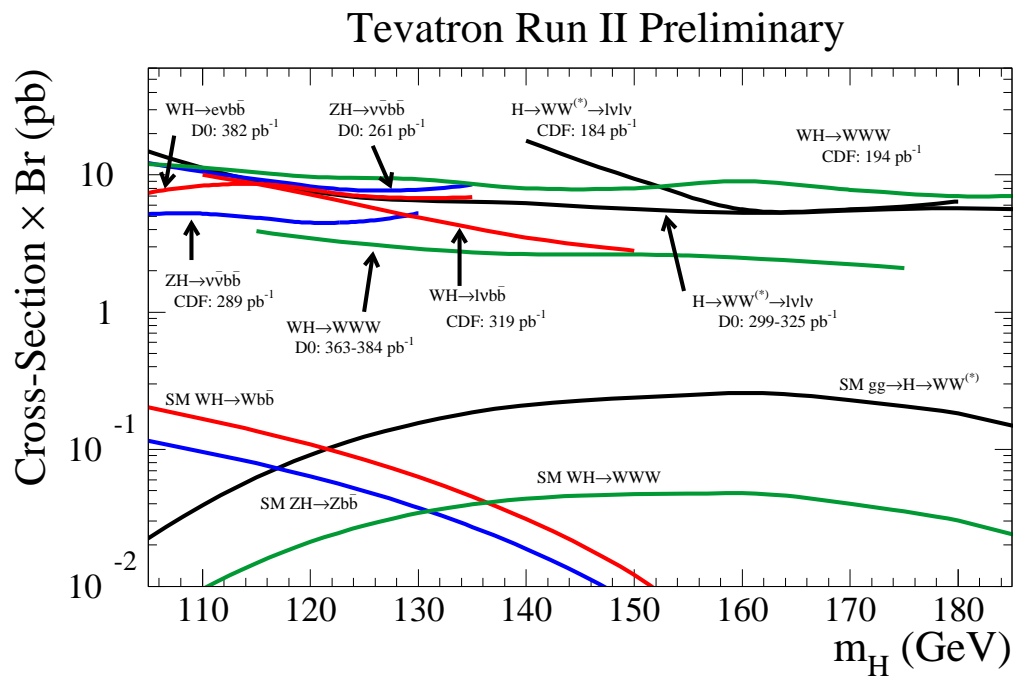

FIGURE 1. Summary of SM Higgs searches at the Tevatron. Summer 2005.

Through this paper, unless otherwise indicated, $95 \%$ C.L. limits will be reported.

\section{FROM THE STANDARD MODEL TO BEYOND}

Although tested to a very good accuracy, the SM is incomplete as the Higgs particle, a cornerstone of the theory, has not been discovered. The best limit for its mass, a free parameter of the theory, $M_{H}>114.4 \mathrm{GeV} / \mathrm{c}^{2}$, is due to the LEP experiments. At this mass the Higgs decays into two $b$ quarks most of the time. At the moment the hunt for Higgs takes place at the Tevatron, where most of the production cross section is via $g g$ fusion $\left(\sigma \simeq 0.8 \mathrm{pb}\right.$ at the LEP limit). At low Higgs masses $\left(M_{H} \leq 130 \div 140 \mathrm{GeV} / \mathrm{c}^{2}\right)$ the signal is swamped by the much larger heavy flavour production. From an experimental point of view, at low mass, the associated production of vector boson ( $W$ or $Z$ ) to Higgs $(\sigma \simeq 0.2 \div 0.1 \mathrm{pb})$ has a clean signature and a better $\mathrm{S} / \mathrm{B}$ ratio.

CDF and D0 searched for the SM Higgs in a large mass range, exploiting different channels. The negative searches can be summarized in a single plot (see fig. 1) where recent results obtained with $300 \div 400 \mathrm{pb}^{-1}$ are shown [4].

\section{SUSY SEARCHES}

The scalar nature of the Higgs particle brings itself some technical problems to the SM. Its mass gets large corrections which must cancel out. Over the years this problem 


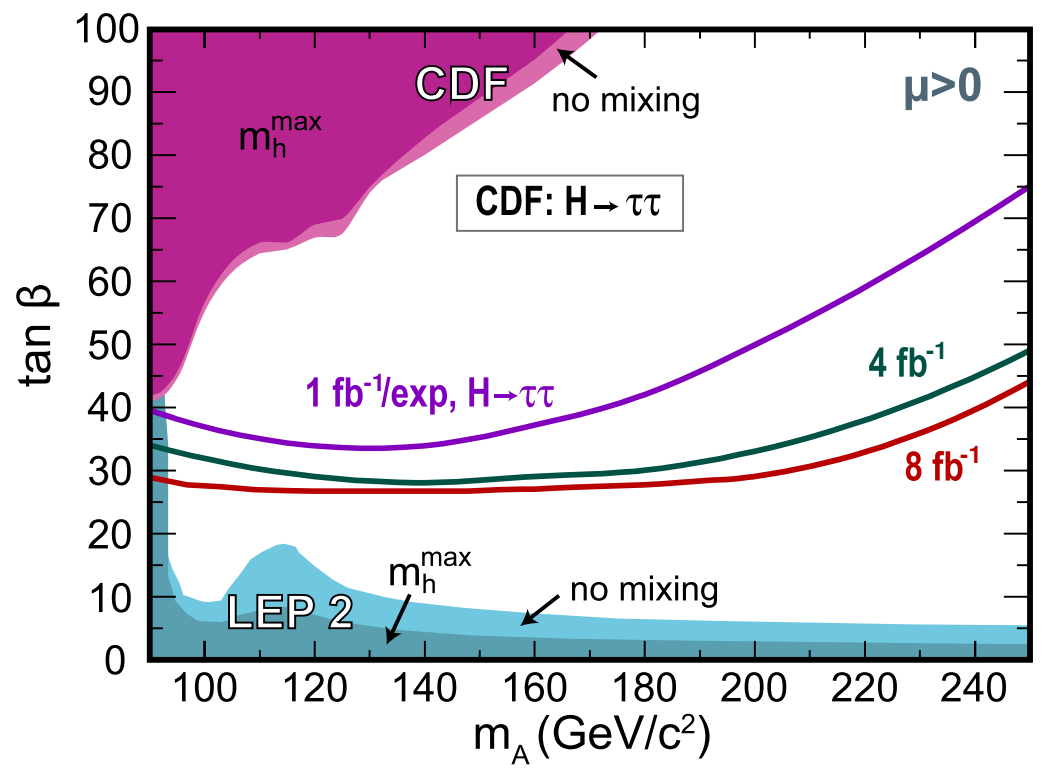

FIGURE 2. CDF limit for MSSM neutral Higgs $A$ (positive $\mu$ case).

has been tackled in many ways, the most popular solution being the SuperSymmetry (SUSY). The idea, borrowed from the particle-antiparticle symmetry, is that there is shadow world of (super)particles. For any fermion a scalar partner would exist, while for each spin 0,1 particle a (s)fermion would exist [3]. Clearly the symmetry is broken, as we do not have seen any sparticle. The nature of SUSY breaking is unknown, and there are several possible scenarios. However, all of them predict an enlarged Higgs sector (the single Higgs doublet of the SM is replaced by two). Also, in most models the assumption is that quantity $R=(-1)^{3(B-L)+2 s}$ is conserved. That implies that in the final state the lightest supersymmetric particle (LSP) will escape detection leaving a typical $v$-like signature of missing energy. SUSY is strongly constrained by the (negative) findings of the LEP experiments. Masses of the sparticle are large therefore currently, the best place to look for SUSY is the Tevatron where typical cross sections are $O(1) \mathrm{pb}$. Due to the unknown nature of the symmetry breaking mechanism, the analyses are (mostly)aimed to specific channels/breaking mechanisms.

\section{MSSM Higgs}

In the minimal supersymmetric model (MSSM), Higgs production can be enhanced, with respect to the SM case, for large $\tan \beta$ (where $\tan \beta$ is the ratio of the v.e.v of the two Higgs doublets). There are five Higgs particles in the model (h, H, A and $H^{ \pm}$), where $h$ and $H$ indicate a light (heavy) neutral Higgs and $\mathrm{A}$ a neutral pseudoscalar. In this model the branching fraction for $H \rightarrow \tau \tau$ channel can be as large as $\simeq 8 \%$. CDF developed a $\tau$ identification algorithm which yielded an $H \rightarrow \tau \tau$ global efficiency of about $0.5 \%$ for the $\tau_{l} \tau h$ decay channel where $l$ indicates an $e$ or $\mu$. Exploiting its dataset of $310 \mathrm{pb}^{-1}$ CDF searched for the $h$ (or $H$ ) and $A$ particle (produced by $g g$ or $q q$ ) decaying into $\tau$ 
TABLE 1. D0 search for chargino in trilepton channels. $l$ indicates an high $P_{T}$ isolated track.

\begin{tabular}{lcccccc}
\hline & $e e l$ & $\mu \mu l$ & $\mu \mu^{*}$ & $e \mu l$ & $\mu \tau l$ & $e \tau l$ \\
\hline Expec.back. & $0.21 \pm 0.12$ & $1.75 \pm 0.57$ & $0.66 \pm 0.37$ & $0.31 \pm 0.13$ & $0.36 \pm 0.13$ & $0.58 \pm 0.14$ \\
Obs. & 0 & 2 & 1 & 0 & 1 & 0 \\
\hline
\end{tabular}

* Same sign muons.

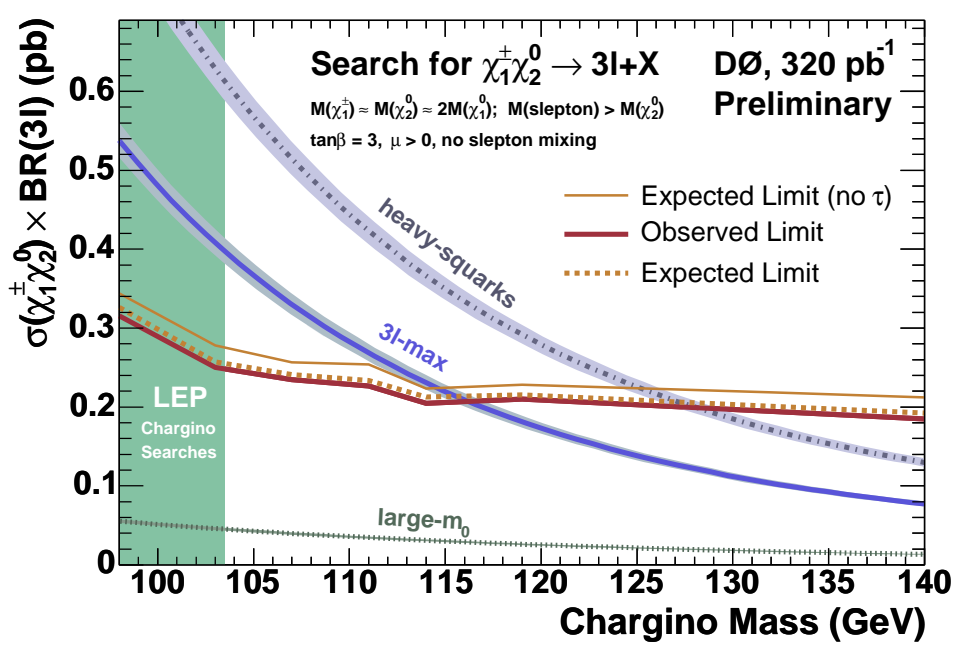

FIGURE 3. D0 limit for chargino mass from trileptons searches.

pairs. They found 236 events where $263 \pm 30.1$ were predicted [5]. The negative search is translated into a limit set within the framework of the MSSM (see fig. 2 2 ). With 1 $\mathrm{fb}^{-1}$ or more, CDF expects to be able to explore the region down to $\tan \beta \simeq 30 \div 40$ for $m_{A}>120 \mathrm{GeV} / \mathrm{c}^{2}$ as shown in fig. 2 .

\section{Chargino and neutralino searches}

Exploiting the large production cross section for chargino and neutralino associated production, both experiments performed several searches. The golden channel is the one in which there are three charged leptons in the final states, associated to the presence of large missing $E_{T}$ (signature of the LSP escaping detection). The low background and easy triggering on this channel is somewhat balanced by the need for good acceptance.

D0 searched into six different channels (see tab. 1) in $320 \mathrm{pb}^{-1}$. SM background is different from channel to channel, mostly due to misendified leptons and to diboson production. The negative result ( 4 events observed where $3.85 \pm 0.75$ are expected) is converted into limits in the framework of a specific mSUGRA scenario (light sleptons, low $m_{0}$ ) and yield a limit for $M_{\chi^{ \pm}}>117 \mathrm{GeV} / \mathrm{c}^{2}$ (see fig. 3)

In Gauge Mediated SUSY Breaking (GMSB) models, gravitino becomes the LSP and neutralino becomes the next LSP (NSLP). Therefore neutralino decays into gravitino$\gamma$. For short neutralino lifetimes the experimental signature is a final state with 2 high 


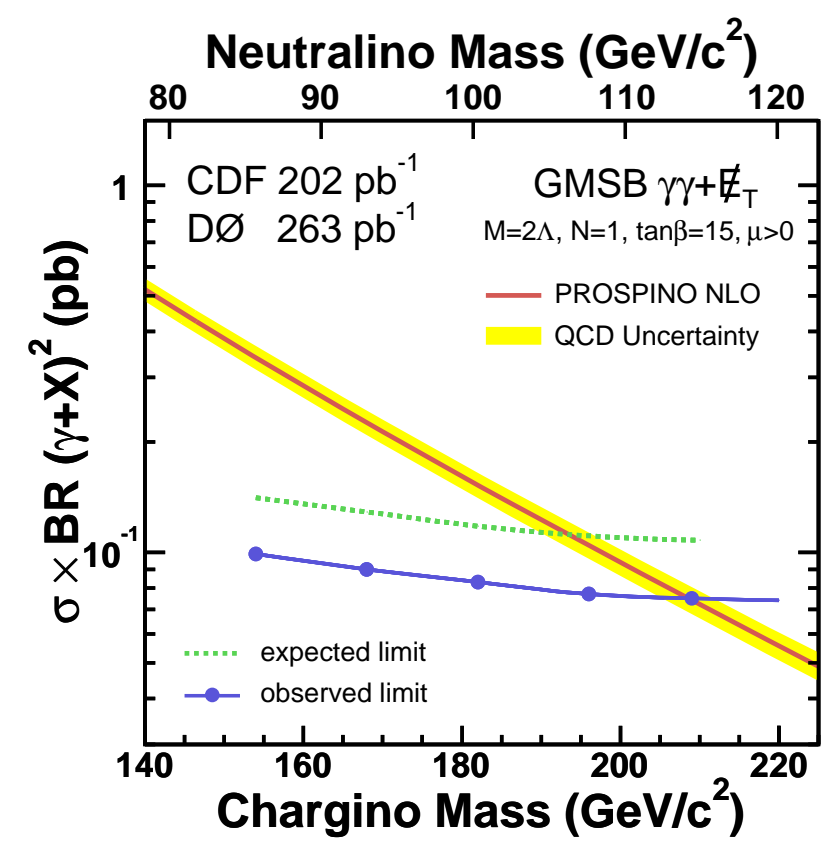

FIGURE 4. Combined CDF/D0 chargino mass limit in GMSB model.

$E_{T} \gamma$ and MET. CDF searched $200 \mathrm{pb}^{-1}$ for events with MET $>45 \mathrm{GeV}$ and $2 \gamma$ s with $E_{T}>13 \mathrm{GeV}$, D0 looked into $263 \mathrm{pb}^{-1}$ for events with MET $>40 \mathrm{GeV}$ and $2 \gamma$ with $E_{T}>20 \mathrm{GeV}$. Both searches gave a negative result, and the combined limit is set at a chargino mass of $209 \mathrm{GeV} / \mathrm{c}^{2}$ (see fig. 4] [6].

\section{Gluino and Squarks}

Decays of $\tilde{g}$ and $\tilde{q}$ at the Tevatron produce multijet events with missing energy. Both experiments looked for events with such topologies. D0 search for $\tilde{g}$ and $\tilde{q}$ was optimized as different regions of the $\tilde{g}-\tilde{q}$ mass plane were explored by using three different subsamples divided according to the number of jets and using $H_{T}$ cut to reduce multijet background. Di-jet events $\left(H_{T}>250 \mathrm{GeV}\right.$ and MET $\left.>175 \mathrm{GeV}\right)$ were selected to search in the region $m_{\tilde{g}} \geq m_{\tilde{q}}$. Three jet events with $H_{T}>325 \mathrm{GeV}$ and MET $>100 \mathrm{GeV}$ to cover the region $m_{\tilde{q}} \sim m_{\tilde{g}}$ and finally four jet events $\left(H_{T}>250 \mathrm{GeV}, \mathrm{MET}>75 \mathrm{GeV}\right)$ to cover the region $m_{\tilde{q}} \gg m_{\tilde{g}}$. After optimizing the selection to reduce multijet background, the final results are the following: 12 events found where $12.8 \pm 5.4$ are expected in dijet sample, 5 events found in three jet sample where $6.1 \pm 3.1$ are expected and 10 events found where $7.1 \pm 0.9$ are expected in four jet sample. Limits are then set within the mSUGRA model for the scenario: $\tan \beta=3, A_{0}=0, \mu<0$ (see fig. 5).

If third generations $\tilde{q}$ are involved, specific final states with heavy flavours can be favoured. Exploiting samples enriched in $b$-jet, limits on the scalar partner of the $b$ quark $(\tilde{b})$ were set by both experiments. CDF searched for $\tilde{g} \rightarrow \tilde{b}$ decays with $\tilde{b} \rightarrow b+L S P$ in $156 \mathrm{pb}^{-1}$ of data. The search, performed looking for a final state with MET and four jets 


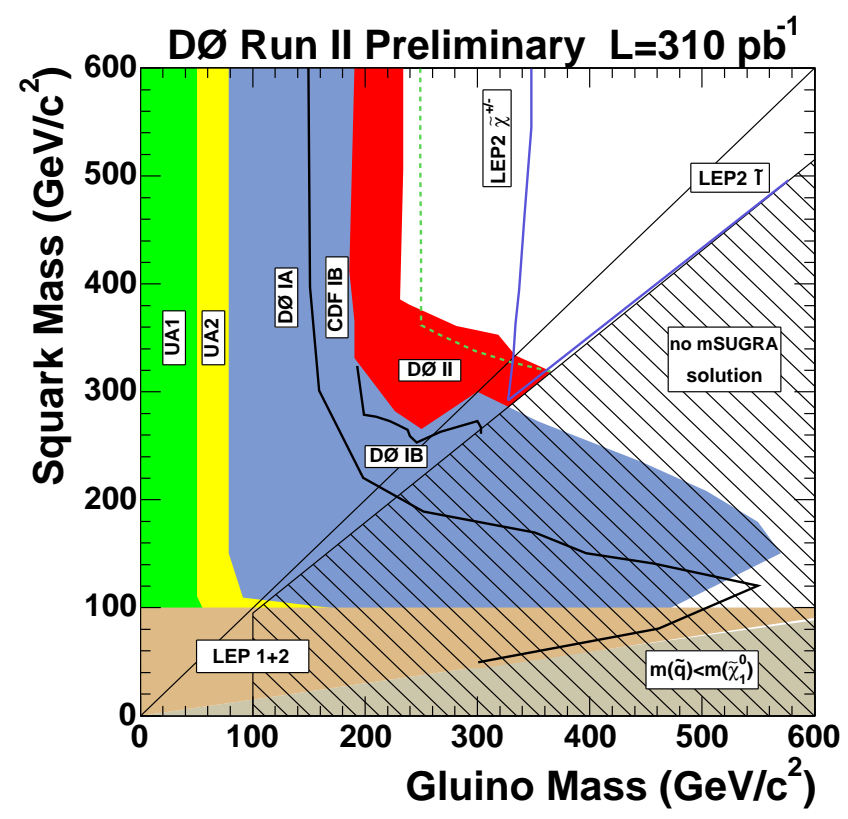

FIGURE 5. Gluino-squark mass limit form D0 searches

(two of them positively identified as containing $b$ quark debris), gave a negative result. The corresponding exclusion plot is shown in fig. 6. D0 searched for signals of direct production of sbottom pairs decaying into $b$ and neutralinos. Using b-tagging to improve $\mathrm{S} / \mathrm{B}$ ratio they are left (in $310 \mathrm{pb}^{-1}$ ) with events consistent with SM expectations and excluded sbottom masses up to $\simeq 200 \mathrm{GeV} / \mathrm{c}^{2}$ for neutralino masses up to $80 \mathrm{GeV} / \mathrm{c}^{2}$.

\section{SUSY indirect searches}

A very important way to test for SUSY is to look at effects on SM processes due to SUSY contributions. A promising channel is $B_{s, d} \rightarrow \mu \mu$ as $B_{s}$ (and $B_{d}$ ) are copiously produced at the Tevatron. SM branching fraction $(\mathrm{BF})$ is $(3.5 \pm 0.9) \times 10^{-9}$ but, in SUSY models, can be enhanced by several order of magnitudes for large $\tan \beta$. CDF and D0 searched in 364 and $300 \mathrm{pb}^{-1}$ of data. CDF set 90(95) \% C.L. limit to $B F\left(B_{s} \rightarrow\right.$ $\mu \mu)<1.5(2) \times 10^{-7}$ and $B F\left(B_{d} \rightarrow \mu \mu\right)<3.8(4.9) \times 10^{-8}$ (better than BaBar limit of $\left.8 \times 10^{-8}\right)$. D0 limit at $90 \%$ C.L. is $B F\left(B_{s} \rightarrow \mu \mu\right)<4.1 \times 10^{-7}$ which, combined with CDF result, yield a Tevatron limit of $1.2 \times 10^{-7}$ at $90 \%$ C.L. Those results set a strong limit on MSSM inspired SUSY models [7]. 


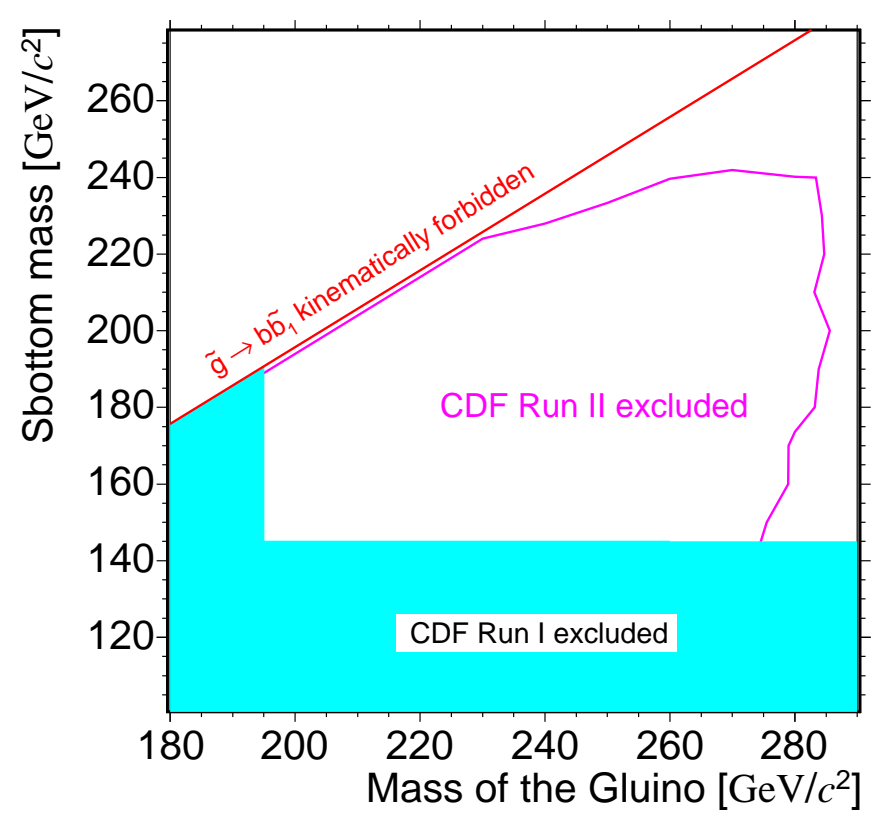

FIGURE 6. CDF limit on $\tilde{g} \rightarrow \tilde{b}$ decays.

\section{RPV SUSY}

In some SUSY theories quantity $R$ is not conserved, that implies that MET (due to escaping LSP) is not a typical signature. The relevant parameter is represented by the couplings among generations. Searches for RPV SUSY take place at both the Tevatron and HERA. At the Tevatron CDF searched, without success, for sneutrino production and set limit to $m_{\tilde{v}}>205 \mathrm{GeV} / \mathrm{c}^{2}$ for the coupling $\lambda_{311}=0.01$. ZEUS and H1 set limits to $\tilde{t}$ production. In fig. 7 you see that for $\lambda \simeq 0.01 M_{\tilde{t}}>260 \mathrm{GeV} / \mathrm{c}^{2}$ at $95 \%$ C.L.

\section{LIVING ON A BRANE}

In recent years, a new paradigm for the physics Beyond the Standard Model emerged. It is linked to the discovery that, given current theoretical and experimental constraints, there is room for large extra-dimensions. The basic idea is that particles are not pointlike objects, but rather extended strings. The main interest of this class of theories is that they look like a very promising way to quantize gravity. Indeed the weakness of gravitation in our world is explained as being the remnant (in standard 3+1 D world) of a stronger interaction which propagates into the extra (hidden) dimensions which are then compactified. There are several options for the mechanism to work. In the Arkani-Hamed, Dimopolous, Dvali (ADD) theory the relevant parameter is the string scale $M_{s}$ which relates the Planck scale to the compatictification radius. In RandallSundrum theories the gravity is localized in the Extra Dimensions (which are highly warped) and the relevant parameters are the compactification radius and the curvature 


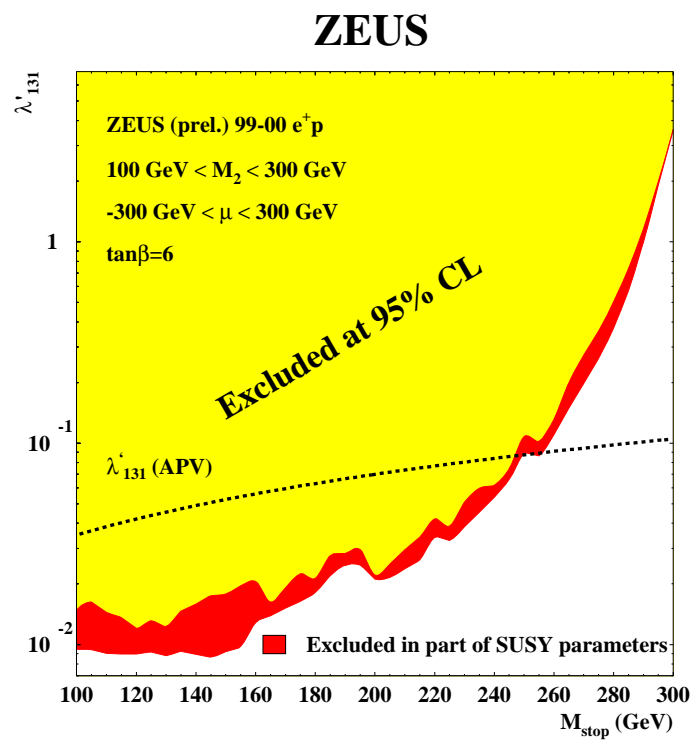

FIGURE 7. ZEUS limit on stop mass in RPV SUSY.

TABLE 2. D0 combined (RunI-II) limits for large extra dimensions.

\begin{tabular}{lccccc}
\hline & GRW & Hewett & HLZ, $\mathbf{n = 2}$ & HLZ, $\mathbf{n = 3}$ & HLZ, $\mathbf{n = 4}$ \\
\hline$e e+\gamma \gamma$ & 1.43 & 1.28 & 1.67 & 1.70 & 1.43 \\
$\mu \mu$ & 1.09 & 0.97 & 1.00 & 1.29 & 1.09 \\
\hline
\end{tabular}

scale thate relate the Planck scale to the $\mathrm{TeV}$ scale. As each particle is seen as part of a tower of Kaluza-Klein excitations, the main phenomenological effect is the existence of such states. At the Tevatron both direct searches for gravitons (RS model) as well as indirect searches of effects on SM processes (ADD model) were performed. In the latter category, $\mathrm{CDF}$ and $\mathrm{D} 0$ searched for modification to $e e, \mu \mu, \gamma \gamma$ production due to graviton exchange. D0 searched in its data by studying its double differential cross section:

$$
\frac{d^{2} \sigma}{d M d \cos \theta^{*}}=f_{S M}+f_{i n t} \eta_{G}+f_{K K} \eta_{G}^{2}
$$

The effect of the existence of extra-dimensions is parametrized (using $f$ and $\eta_{G}$ ) according to different theoretical assumption. Data is consistent with SM expectations and limits are set in different scenarions. In table 2 we show a summary of the results in various channels according to several theoretical options (Giudice-Rattazzi-Wells, Hewett, Han-Lykken-Zhang)

In direct searches, both experiments looked for signal of direct production of the Randall-Sundrum graviton (a spin 2 particle) in $\gamma \gamma$ and dilepton (e and $\mu$ ) samples. CDF analized $345 \mathrm{pb}^{-1}$ of data and D0 looked at $\simeq 250 \mathrm{pb}^{-1}$ of data. Results are consistent with SM expectations and limits are set. The strongest constraint is obtained by D0 that, for $k / M_{P l}=0.1(0.01)$ set a $95 \%$ C.L. limit of $M_{G}>785(250) \mathrm{GeV} / \mathrm{c}^{2}$ (fig. 8). 


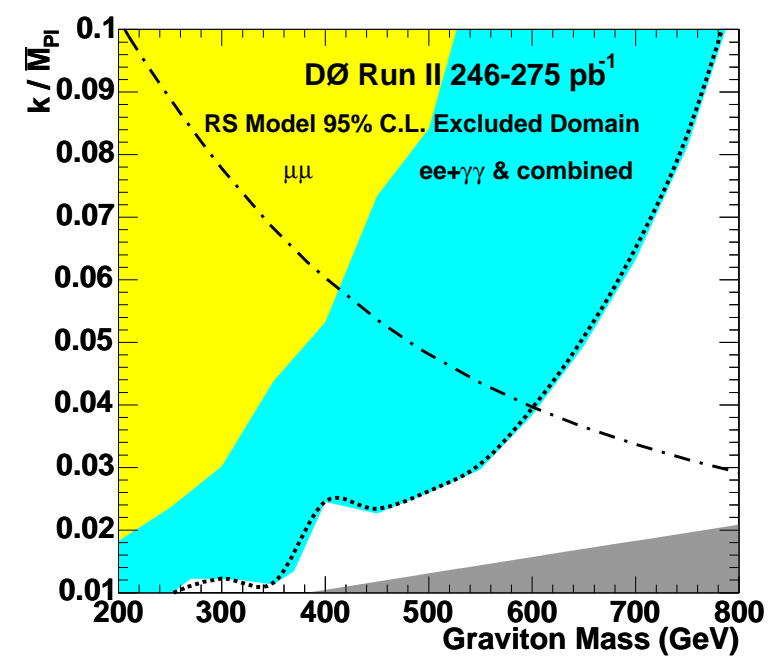

FIGURE 8. D0 limit on the mass of R.-S. graviton.

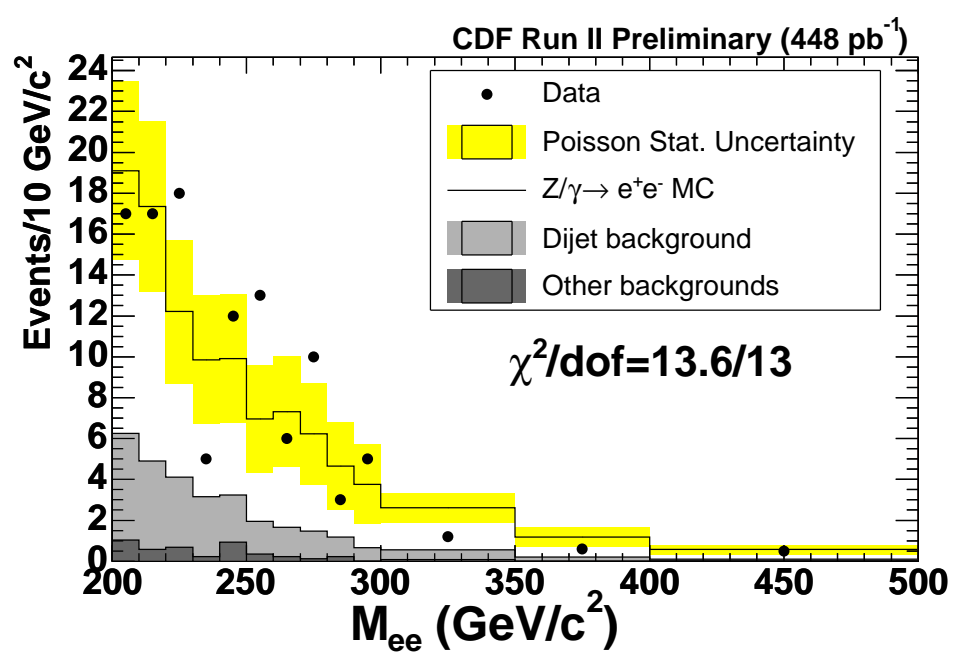

FIGURE 9. CDF Zp

\section{SEARCHING IN THE DILEPTON SAMPLE}

The dilepton channels have been through investigated by both experiments as any deviation from SM would easily signal new physics. The measured quantity is $\sigma \times B F$, and negative findings can be interpreted as limits for physical states by selecting a specific theoretical model. Indeed, while the acceptance has strong dependence mainly from the spin of the mother particle, an important role is played by the (unknown) couplings. CDF recentlty presented a preliminary analysis of data in the ee channel. In fig. 9 the search region $\left(M_{e e}>200 \mathrm{GeV} / \mathrm{c}^{2}\right)$ is shown. No visible excess over SM expectations is seen in $448 \mathrm{pb}^{-1}$. In order to convert negative findings into limits, modelspecific calculations are performed. Also, a standard way to estimate the sensitivity to a 
TABLE 3. Limit for a sequential $Z$. CDF limits for $E_{6} Z^{\prime}$ refer to $e e$ and $\mu \mu$ channel combined using $200 \mathrm{pb}^{-1}$.

\begin{tabular}{lccccccc}
\hline & $\mathbf{S M}(e e)$ & $\mathbf{S M}(\mu \mu)$ & $\mathbf{S M}(\tau \tau)$ & $Z_{I}$ & $Z_{\chi}$ & $Z_{\psi}$ & $Z_{\eta}$ \\
\hline $\mathrm{CDF}$ & 845 & 735 & 394 & 610 & 670 & 690 & 715 \\
$\mathrm{D} 0$ & 780 & 680 & - & 575 & 640 & 650 & 680 \\
\hline
\end{tabular}

$Z$-like particle is to assume SM couplings. The CDF result in $448 \mathrm{pb}^{-1}$ converts into a limit on SM-like $Z^{\prime}, M_{Z^{\prime}}>845$ at $95 \%$ C.L. In table 3 we show the summary of searches in different decay channels.

D0 also analized its dilepton samples (ee and $\mu \mu$ ) looking for effects due to preons, the putative elementary constituents of quarks and leptons. The effects are parametrized in terms of $\Lambda$, the compositness scale. As for the LED case, the search compared the spectrum invariant mass and scattering angle of data with SM expectations. The parametrization used is:

$$
\frac{d^{2} \sigma}{d M d \cos \theta^{*}}=f_{S M}+\frac{I}{\Lambda^{2}}+\frac{C}{\Lambda^{4}}
$$

where $I$ and $C$ are input from theory and express the possibility of constructive/destructive interference. The negative result is used to set limits on the fundamental scale $\Lambda$. Limit is $\Lambda>3.6(9.1) \mathrm{TeV}$ for constructive(destructive) interference in the electron channel in $271 \mathrm{pb}^{-1}$. In the muon channel, using $400 \mathrm{pb}^{-1}$ the limit is stronger and set to $\Lambda>4.2(9.8) \mathrm{TeV}$ for constructive(destructive) interference.

\section{LEPTOQUARKS}

The fermionic sector of the SM can be symmetrized by the introduction of still unknown particles -LeptoQuarks (LQ)- carrying both barionic and leptonic numbers and coupling to both quarks and leptons. Relevant parameters for the theory are the couplings, $\lambda_{i j}$ and the decays branching fractions, which are ordered according to the BF into charged leptons $(\beta)$ which can vary between 1 and 0 .

At HERA first generation LQs can be produced in resonant process. Both $\mathrm{H} 1$ and ZEUS searched for signals of LQ production in their CC and NC samples. No signal is found and limits are set. Due to the way LQs are produced those limits are a function of the LQ mass and of the couplings. In fig. 10 we show the result from $\mathrm{H} 1$ which, for a coupling strength $\simeq 0.3$ is $M_{L Q}>290 \mathrm{GeV} / \mathrm{c}^{2}$.

At the Tevatron both experiments searched for scalar LQs in all generations. As LQs are pair-produced, the actual measurement can be one of the following: $\sigma \times \beta^{2}$, $2 \times \sigma \beta(1-\beta), \sigma \times(1-\beta)^{2}$, depending upon the BF into charged leptons. The latter channel has a final state of two jets and MET and the analysis performed is generationindependent. CDF report a negative result in this channel and set a limit, using $191 \mathrm{pb}^{-1}$, to $M_{L Q}>117 \mathrm{GeV} / \mathrm{c}^{2}$. More interesting are the results for first and second generation LQ. In those cases both experiments analized their samples $l l j j, l v j j, v v j j$ where $l=e, \mu$. CDF reports a negative result and set limits, for $\beta=1$ to $M_{L Q}>235(224)$ 


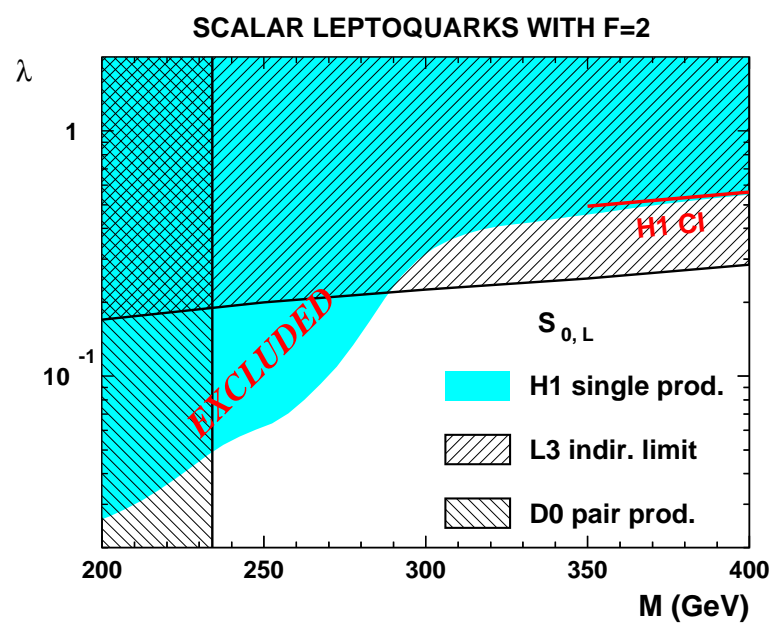

FIGURE 10. H1 limit for LQ production.

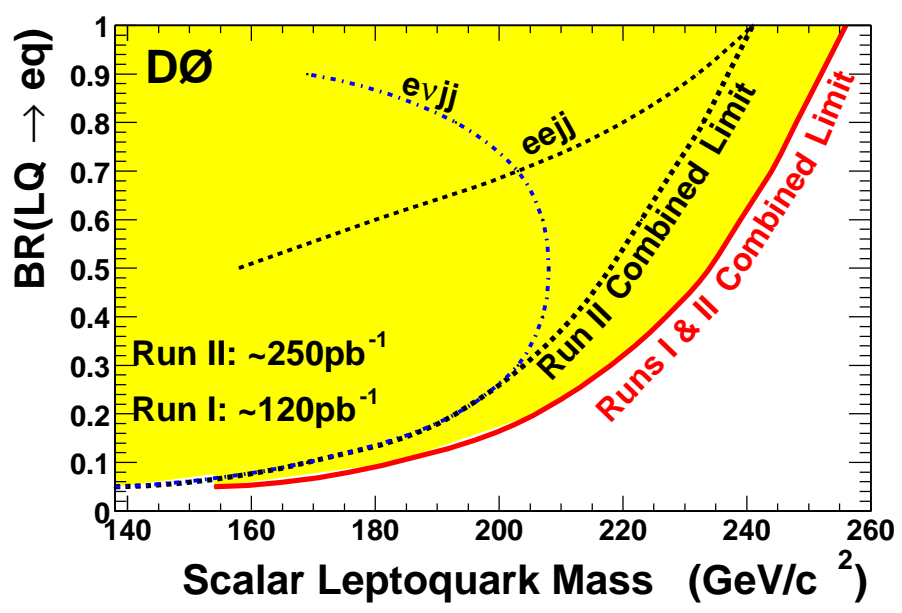

FIGURE 11. D0 limits for first generation LQ.

$\mathrm{GeV} / \mathrm{c}^{2}$ for $1^{s t}\left(2^{\text {nd }}\right)$ generation LQ in $200 \mathrm{pb}^{-1}$. D0 combines its negative results in 250 $\mathrm{pb}^{-1}\left(1^{\text {st }}\right)$ and $294 \mathrm{pb}^{-1}\left(2^{\text {nd }}\right)$ generation with Run I results and set limits for $\beta=1$ to $M_{L Q}>256(251) \mathrm{GeV} / \mathrm{c}^{2}$ for $1^{s t}\left(2^{n d}\right)$ generation LQ (see fig. 11, 12).

CDF searched for direct production of $3 r d$ generation LQ in an analysis which also set limits on RPV SUSY stop production. As $4.8 \pm 0.7$ events are found in $200 \mathrm{pb}^{-1}$, where 5 are expected a limit is set $M_{L Q}>129 \mathrm{GeV} / \mathrm{c}^{2}$. 


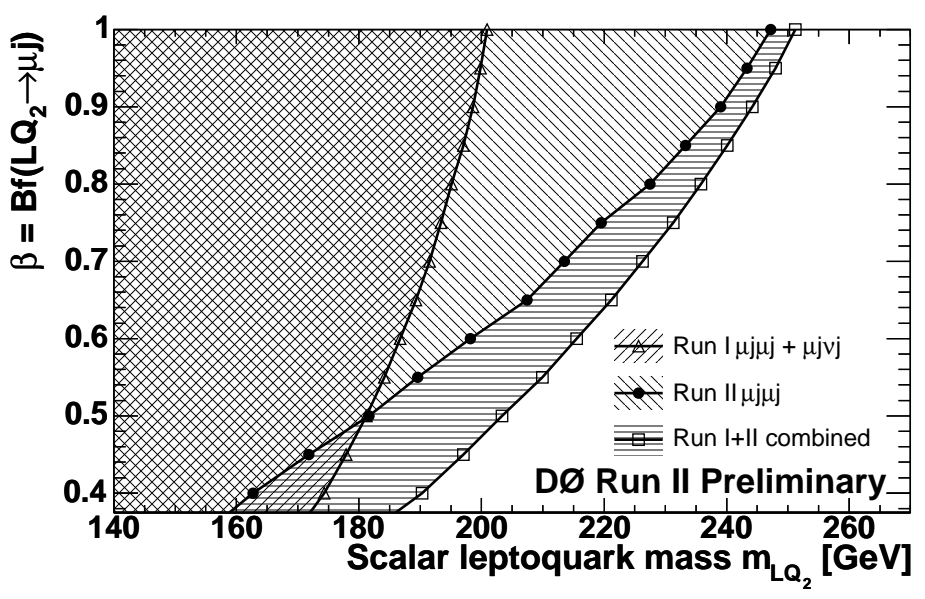

FIGURE 12. D0 limist for second generation LQ.

\section{CONCLUSION}

A number of searches for signals of physics beyond the SM are ongoing. While their current findings do not support evidence of such processes, a very exciting period is ahead of us. The expectations of CDF and D0 to collect 4 to $8 \mathrm{fb}^{-1}$ per experiment by 2009, as well as the LHC startup, indicate that within a few years either we will have seen signal of non-SM physics, or a number of theories will be disproved.

\section{ACKNOWLEDGMENTS}

I would like to thanks my colleagues from the Tevatron and HERA for the preparation of this talk, and in particular B.Heinemann, S.Ming Wang, J.F.Grivaz, M.Wing, E.Tassi, E.Gallo, A.Anastassov.

\section{REFERENCES}

1. See presentations by J. Terron and Z. Zhang, these Proceedings.

2. For the status of D0 and CDF see R. Erbacher and P. Petroff, these Proceedings.

3. H. E. Haber and G. L. Kana, Phys.Rept117,75 (1985)

4. Most recent results for this and other analyses from CDF can be found at http://www-cdf.fnal.gov/physics/exotics/exotics.html and from D0 at: http://www-d0.fnal.gov/Run2Physics/WWW/results/np.htm

5. A. Abulencia et al., CDF Collaboration, hep-ex/050851.

6. V. Buescher et al. hep-ex/0504004

7. See for example J. Ellis et al. Phys.Lett.B 624, 47 (2005). 